\title{
Dynamic characteristics of the exciton and the biexciton in a single InGaN quantum dot
}

Supaluck Amloy, Evgenii Moskalenko, M Eriksson, K Fredrik Karlsson, Y T Chen, K H Chen, H C Hsu, C L Hsiao, L C Chen and Per-Olof Holtz

\section{Linköping University Post Print}

N.B.: When citing this work, cite the original article.

Original Publication:

Supaluck Amloy, Evgenii Moskalenko, M Eriksson, K Fredrik Karlsson, Y T Chen, K H Chen, H C Hsu, C L Hsiao, L C Chen and Per-Olof Holtz, Dynamic characteristics of the exciton and the biexciton in a single InGaN quantum dot, 2012, Applied Physics Letters, (101), 6.

http://dx.doi.org/10.1063/1.4742343

Copyright: American Institute of Physics (AIP) http://www.aip.org/

Postprint available at: Linköping University Electronic Press http://urn.kb.se/resolve?urn=urn:nbn:se:liu:diva-84908 


\title{
Dynamic characteristics of the exciton and the biexciton in a single InGaN quantum dot
}

\author{
S. Amloy, ${ }^{1,2, a)}$ E. S. Moskalenko, ${ }^{1,3, b)}$ M. Eriksson, ${ }^{1}$ K. F. Karlsson, ${ }^{1}$ Y. T. Chen, ${ }^{4}$ \\ K. H. Chen, ${ }^{4,5}$ H. C. Hsu, ${ }^{5}$ C. L. Hsiao, ${ }^{5}$ L. C. Chen, ${ }^{5}$ and P. O. Holtz ${ }^{1}$ \\ ${ }^{1}$ Department of Physics, Chemistry, and Biology (IFM), Linköping University, S-58183 Linköping, Sweden \\ ${ }^{2}$ Department of Physics, Faculty of Science, Thaksin University, Phattalung 93110, Thailand \\ ${ }^{3}$ A. F. Ioffe Physical-Technical Institute, Russian Academy of Sciences, 194021, Polytechnicheskaya 26, \\ St. Petersburg, Russia \\ ${ }^{4}$ Institute of Atomic and Molecular Sciences, Academia Sinica, Taipei 106, Taiwan \\ ${ }^{5}$ Center for Condensed Matter Sciences, National Taiwan University, Taipei 106, Taiwan
}

(Received 16 May 2012; accepted 20 July 2012; published online 7 August 2012)

\begin{abstract}
The dynamics of the exciton and the biexciton related emission from a single InGaN quantum dot (QD) have been measured by time-resolved microphotoluminescence spectroscopy. An exciton-biexciton pair of the same QD was identified by the combination of power dependence and polarization-resolved spectroscopy. Moreover, the spectral temperature evolution was utilized in order to distinguish the biexciton from a trion. Both the exciton and the biexciton related emission reveal mono-exponential decays corresponding to time constants of $\sim 900$ and $\sim 500 \mathrm{ps,} \mathrm{respectively.}$ The obtained lifetime ratio of $\sim 1.8$ indicates that the QD is small, with a size comparable to the exciton Bohr radius. (C) 2012 American Institute of Physics. [http://dx.doi.org/10.1063/1.4742343]
\end{abstract}

Quantum dots (QDs) are especially attractive as sources of single ${ }^{1,2}$ and correlated photons ${ }^{3,4}$ for quantum information processing ${ }^{5,6}$ and quantum computing applications. ${ }^{7,8}$ Most studies on QDs are based on III-arsenide materials, and only a few investigations have so far been reported for the III-nitride materials. However, the optically efficient IIInitride material is tunable over a wider range of band gaps than the arsenides, with the consequence that it enables heterostructures with deeper confinement. This characteristic has an attractive potential for room temperature operation of QD based quantum devices. ${ }^{2}$

A basic understanding of the exciton and the biexciton related emissions from single QDs is required in order to fully exploit their application potential. The biexciton binding energy of GaN QDs was recently found to scale with the dot size ${ }^{9}$ and to exhibit both positive and negative values. ${ }^{10}$ This opens for the possibility to obtain zero biexciton binding energy and thereby generate quantum entangled photon pairs in a cascade recombination of the biexciton. ${ }^{11}$ In addition, measurements have revealed a strong polarization anisotropy of the photons emitted in the radiative decay of the excitons and the biexcitons for nitride based QDs, ${ }^{12-14}$ which is attributed to the asymmetry of the confinement potential. However, typically, no clear evidence of the expected polarized fine structure splitting (FSS) can be observed in the standard measurements geometry with the light extracted from the c-axis, ${ }^{12,15}$ but recently fine structure splittings of $100-340 \mu \mathrm{eV}$ could be resolved for InGaN QDs using a sideview geometry. ${ }^{16}$

The photon generation rate is determined by the spontaneous radiative recombination lifetimes of the exciton and the biexciton. Excitonic recombination lifetimes for (In) GaN

\footnotetext{
${ }^{\text {a) }}$ Author to whom correspondence should be addressed. Electronic mail: supaluck@ifm.liu.se.

${ }^{\text {b) Deceased. }}$
}

QDs have been reported to be in the wide range of 0.4$180 \mu \mathrm{s} .{ }^{17-19}$ The huge variation of the measured lifetimes is related to the electron and hole wave function overlap, affected by the dot size and shape, as well as the indium concentration in the QDs and their surroundings. ${ }^{17}$ These results are supported by theoretical results which show that the built-in piezo- and pyroelectric fields within the InGaN/GaN QDs cause a sensitive dependence of the radiative lifetime on the QD geometry and composition. ${ }^{20}$ To date, merely a few studies compare the exciton and the biexciton dynamics of a single InGaN QD, with the lifetime of the biexciton found to be either approximately the same as that of the exciton $^{12,21}$ or significantly longer than that of the exciton. ${ }^{22}$

In order to study the recombination lifetimes of both the exciton and the biexciton belonging to the same QD, a reliable spectral identification is required. The expected linear and quadratic excitation power dependences of the photoluminescence (PL) intensities of the exciton and the biexciton, ${ }^{23}$ respectively, are not sufficient to guarantee that the emissions originate from the same QD. Moreover, it has been demonstrated that also a trion under certain conditions can exhibit a quadratic power dependence. ${ }^{24}$

In this work, the exciton and the biexciton dynamics of a single InGaN QD are studied. In addition to the conventional power dependence, spectral identification of the exciton and the biexciton relies on polarization resolved spectroscopy ${ }^{13}$ as well as temperature dependence. In contrast to previous works on III-nitride QDs, it is found that the lifetime of the biexciton is about 1.8 times shorter than that of the exciton, i.e., a result similar to typical InGaAs QDs.

The InGaN QDs sample investigated in this work was grown on a c-plane sapphire substrate at the temperature of $500^{\circ} \mathrm{C}$ by means of plasma-assisted molecular beam epitaxy. A $\sim 30 \mathrm{~nm} \mathrm{GaN} \mathrm{buffer} \mathrm{layer} \mathrm{was} \mathrm{grown} \mathrm{on} \mathrm{the} \mathrm{substrate,}$ followed by a $\sim 2.5 \mathrm{~nm}$ InGaN quantum well (QW). InGaN QDs were formed from this QW layer with an In composition 
of nominally $20 \%$ which finally was capped with a $\sim 30 \mathrm{~nm}$ GaN layer. The wurtzite crystallization of the $\mathrm{InGaN}$ layer was confirmed by $\mathrm{x}$-ray diffraction (XRD) measurements. The density of optically active QDs was determined to be about $\sim 10^{9}-10^{10} \mathrm{~cm}^{-2}$ as determined from the number of sharp PL emission lines. ${ }^{16}$

Microphotoluminescence $(\mu \mathrm{PL})$ spectra of single dots were measured through circular apertures (diameter of $260 \mathrm{~nm}$ ) in a thin aluminum layer coated on the sample surface. A refractive objective lens focused a continuous-wave $355 \mathrm{~nm}$ excitation laser, with a high spatial resolving power of $\sim 1 \mu \mathrm{m}$, onto the sample surface. The sample was mounted on a cold finger inside a cryostat, which was cooled down to $4 \mathrm{~K}$ by a continuous flow of liquid helium. The PL signal was collected by the same objective lens and dispersed by a $0.55 \mathrm{~m}$ focal length monochromator with 1200 grooves $/ \mathrm{mm}$, giving a spectral resolution $\sim 2 \mathrm{meV}$ when detected by a liquid nitrogen cooled charge-coupled device (CCD) detector. The linear polarization of the PL emission was analyzed by a rotatable half-wave retardation plate and a fixed linear polarizer placed in the signal path in front of the monochromator. The polarization dependence was determined by fitting the experimental PL intensity data $(I)$ with the formula $I(\theta)=I_{\max } \cos ^{2}(\theta-\varphi)+I_{\min } \sin ^{2}(\theta-\varphi)$, where $I_{\max }$ and $I_{\min }$ are the maximum and minimum intensities, $\theta$ corresponds to the transmission angle of the polarization analyzer, and $\varphi$ is the polarization angle corresponding to maximum intensity. Furthermore, the QDs emission was also measured by timeresolved $\mu \mathrm{PL}$ by using a frequency tripled titanium sapphire excitation laser operating at a wavelength of $266 \mathrm{~nm}$ with a pulse length of $0.5 \mathrm{ps}$ and a repetition rate of $75 \mathrm{MHz}$. The PL signal was dispersed by a monochromator with 150 grooves $/ \mathrm{mm}$ and the recorded PL decay times were acquired by a CCD connected to the photocathode of a streak camera (Hamamatsu C5680).

The $\mu \mathrm{PL}$ power dependence of the studied InGaN QD is shown in Fig. 1(a), with two predominant peaks labeled $X$ and XX. The peaks blueshift by less than $1.5 \mathrm{meV}$ as the excitation power is increased by two orders of magnitude to its maximum value of $110 \mu \mathrm{W}$, evidencing a very small screen-
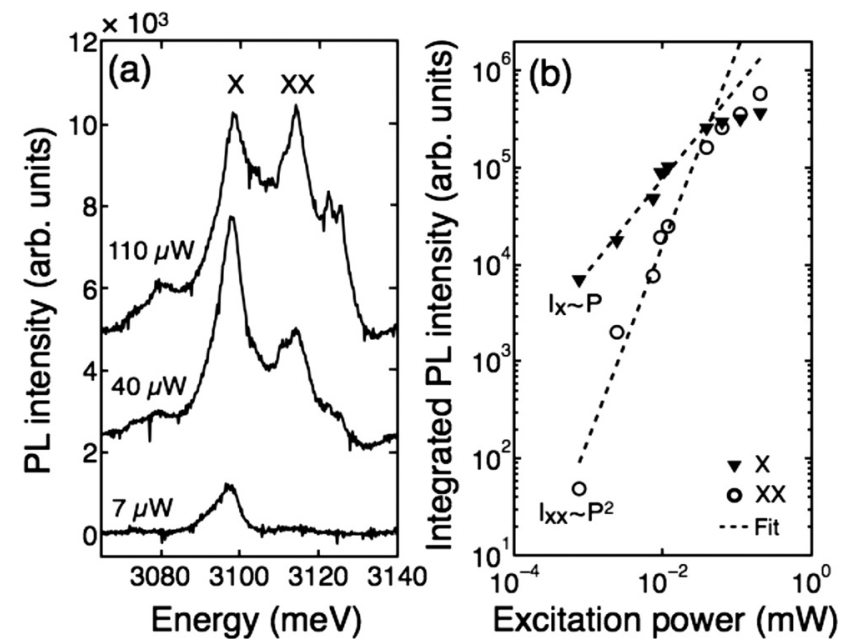

FIG. 1. (a) $\mu \mathrm{PL}$ spectra measured for different excitation powers, as indicated in the figure. (b) The integrated intensities of peaks X and XX plotted as a function of the excitation power. ing effect of the internal electric field by the photoexcited carriers. Moreover, the integrated PL intensity of peak X develops with a linear power dependence while peak XX appears with a quadratic dependence, up to a certain saturation regime (see Fig. 1(b)). Polarization-resolved spectra obtained for different transmission angles of the polarization analyzer are shown in Fig. 2(a). Note that no excitonic finestructure splitting could be resolved, as usual for this top view measurement geometry. ${ }^{12,15}$ Moreover, both peaks X and XX exhibit maximal intensities for the same polarization angle $\varphi \approx 90^{\circ}$ and they display a similar degree of linear polarization $P_{X} \approx 0.50$ and $P_{X X} \approx 0.42$, respectively (see Fig. 2(b)), as evaluated from $P=\left(I_{\max }-I_{\min }\right) /\left(I_{\max }+I_{\min }\right)$. Fig. 2 also includes the polarization dependence of another peak $\mathrm{X}_{0}$, which exhibits a polarization angle $\varphi \approx 130^{\circ}$ and $P \approx 0.56$, different from the peaks $\mathrm{X}$ and $\mathrm{XX}$ (see Fig. 2(b)). In general, the polarization angles of the QDs in the investigated sample appear randomly between different dots, with varying values of $P$ ranging from 0.4 to 0.9 . However, all emission lines originating from the same confinement potential with the hole(s) in the ground state are expected to exhibit identical polarization angle and similar degree of polarization. ${ }^{13}$ It is therefore most likely that peaks $\mathrm{X}$ and $\mathrm{XX}$ originate from the same $\mathrm{QD}$, while $\mathrm{X}_{0}$ is attributed to an excitonic emission from another dot. Thus, it can be concluded that peak X corresponds to the exciton and peak XX is related to an exciton complex involving more carriers than a single electron-hole pair, ${ }^{13,23}$ with a negative binding energy of $-15.8 \mathrm{meV}$. The most probable candidate for peak $\mathrm{XX}$ is the biexciton, for which a quadratic power dependence is expected. ${ }^{23}$ However, a recent study demonstrates that also a trion can exhibit a quadratic dependence under certain conditions. ${ }^{24}$

In order to make a more trustworthy identification of peak XX, the spectral evolution at elevated temperatures was analyzed. The trion related emission is known to compete with the intensity of $X$ as the excitation conditions such as the temperature or excitation power is changed, ${ }^{24-26}$ while

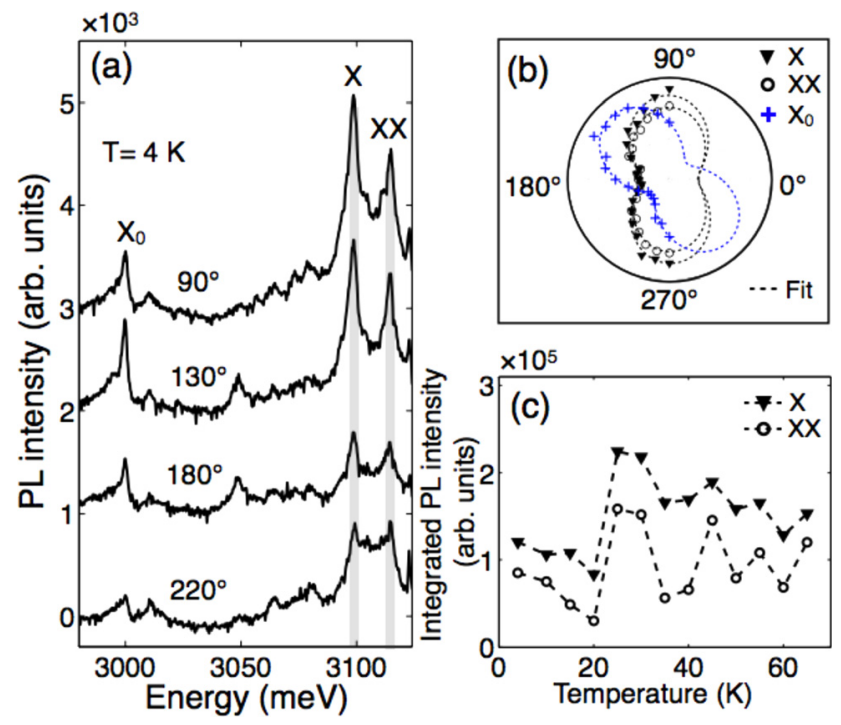

FIG. 2. (a) Polarization resolved $\mu \mathrm{PL}$ spectra obtained for different transmission angles of the polarization analyzer, as indicated in figure. (b) The polar plots of integrated PL intensities of peaks $X$ and $X X$ and $X_{0}$. (c) The integrated PL intensity of peaks $\mathrm{X}$ and $\mathrm{XX}$ versus the temperature. 
such a competition does not occur for the biexciton below the saturation regime. The integrated intensities of peaks $\mathrm{X}$ and $\mathrm{XX}$ for temperatures ranging from 4 to $65 \mathrm{~K}$ are shown in Fig. 2(c). No obvious transfers of intensity from $X$ to $X X$ can be revealed, instead the small changes observed in the intensities of $\mathrm{X}$ and $\mathrm{XX}$ closely resemble each other. This fact strongly suggests that peak XX originates from the biexciton rather than the trion.

Time-resolved PL spectroscopy performed on QD ensembles reveals excitonic lifetimes varying from $\sim 3.0$ down to $\sim 0.8 \mathrm{~ns}$ with increasing emission energy from 3.0 to $3.2 \mathrm{eV}$. Having identified the two main spectral features of a single InGaN QD, we now turn the attention to their individual time evolutions. The time-resolved $\mu \mathrm{PL}$ spectra of the exciton (peak X) and the biexciton (peak XX) both exhibit a mono-exponential decay behavior (see Figs. 3(a) and 3(b)) and, hence, can be well-fitted with the following equation:

$$
I(t)=A \exp \left(-\frac{t}{\tau}\right)
$$

where $I(t)$ is the PL intensity as a function of the time $t, A$ is intensity for $t=0$, and $\tau$ is the recombination lifetime. This exponential model results in a lifetime $\tau_{\mathrm{x}} \approx 880 \mathrm{ps}$ for the exciton and $\tau_{\mathrm{xx}} \approx 500 \mathrm{ps}$ for the biexciton. The mono-exponential behavior as well as the relatively long lifetimes suggest that the obtained values correspond to the radiative lifetimes. The obtained lifetimes are within the span of lifetimes reported previously for single InGaN QDs exhibiting both the excitons and the biexcitons (e.g., $\tau_{\mathrm{x}} \approx 1.0 \mathrm{~ns}, \tau_{\mathrm{xx}} \approx 1.4 \mathrm{~ns}$ (Ref. 22) and $\tau_{\mathrm{x}} \approx 250 \mathrm{ps}, \tau_{\mathrm{xx}} \approx 220 \mathrm{ps}$ (Ref. 12)). As already mentioned, this span reflects the variation of composition and size for the QDs and its barriers. ${ }^{20}$ More notable is the ratio $\tau_{\mathrm{x}} / \tau_{\mathrm{xx}} \approx 1.8$ in this work, while others report significantly smaller values $\sim 0.7$ (Ref. 22) and $\sim 1.0{ }^{12,21}$ The recombination lifetimes of the biexcitons were analyzed by Bacher et al., ${ }^{27}$ for QDs with heavy hole masses, for which the holes move slower and possess more localized wave function than the electrons. The smaller electron effective mass, on the other hand, results in broader wave functions extending throughout the QD. When the size of the QD decreases, the spatial separation of the holes in the biexciton is reduced more than the separation of the
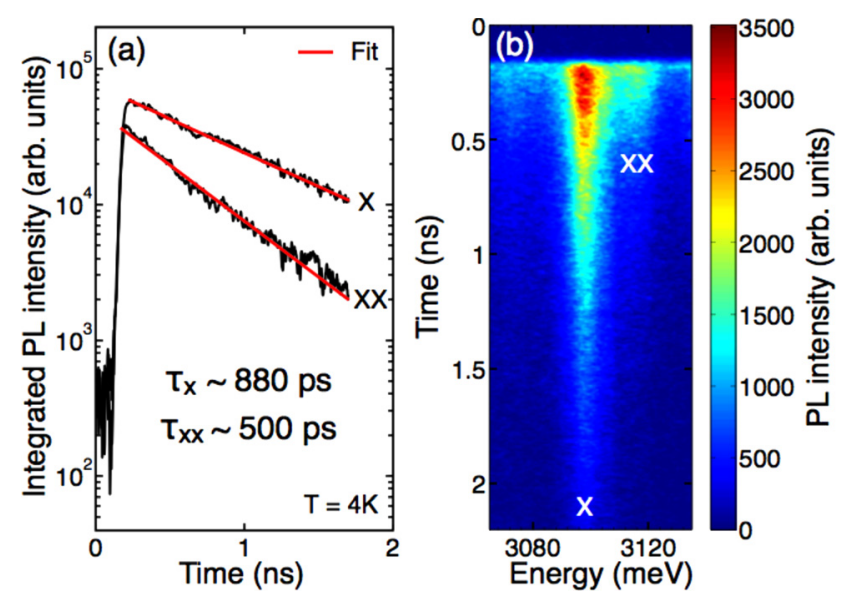

FIG. 3. (a) Time-resolved $\mu \mathrm{PL}$ spectra at $\mathrm{T}=4 \mathrm{~K}$ of peaks $\mathrm{X}$ and $\mathrm{XX}$, with fitted lines according to Eq. (1). (b) Time-resolved $\mu \mathrm{PL}$ image showing the time evolution of the PL spectrum. electrons which are well distributed in the QD, leading to significantly shorter biexciton radiative lifetimes. On the other hand, the exciton lifetime is less sensitive to the QD size due to the lack of hole-hole interactions. Theoretical calculations done for disk shaped QDs with all dimensions less than the exciton Bohr radius ( $a_{\mathrm{Bohr}}$ ) yield the ratio $\tau_{\mathrm{x}} / \tau_{\mathrm{xx}} \approx 2$, while the increase of one lateral dimension to $3 a_{\mathrm{Bohr}}$ reduced $\tau_{\mathrm{x}} / \tau_{\mathrm{xx}}$ $\approx 1.4$ (Ref. 27). Hence, our result $\tau_{\mathrm{x}} / \tau_{\mathrm{xx}} \approx 1.8$ indicates that the investigated single InGaN QD is relatively small, with a size close to the InGaN exciton Bohr radius $\left(a_{\text {Bohr }} \approx 3 \mathrm{~nm}\right.$ (Ref. 28)). This result is supported by the small energy shift of merely $1.5 \mathrm{meV}$ observed for $\mathrm{X}$ in the power dependence suggesting an almost negligible quantum confined Stark effect (QCSE) in the investigated QD. The QCSE depends essentially on the QD height and is known to be small for thin quantum structures (say $1-2 \mathrm{~nm}$ thick $^{29,30}$ ). Our result is also consistent with previous polarization measurements performed on the cleaved-edge on other QDs in the same sample, which revealed a small lateral extension of the QDs based on a significant linear polarization of the excitonic emission along the c-axis. ${ }^{16}$ Moreover, the lateral QD size was estimated to be in the range of $1-5 \mathrm{~nm}$ from scanning transmission electron microscopy images of the studied sample. ${ }^{16}$ Note that the ratio of $\tau_{\mathrm{x}} / \tau_{\mathrm{xx}}$ obtained here in an InGaN QD well corresponds to the typical values obtained for InAs QDs. ${ }^{31,32}$

In conclusion, the exciton and the biexciton were spectrally identified for a single InGaN QD and their dynamical properties were investigated. It was found that both the exciton and the biexciton exhibit a mono-exponential decay behavior with well defined lifetimes of $\sim 900$ and $\sim 500 \mathrm{ps}$, respectively. Unlike previous reports on InGaN QDs, the biexciton lifetime is about half that of the exciton, which can be inferred to the small vertical and lateral extensions of the studied QD, comparable to the Bohr radius of $3 \mathrm{~nm}$.

This work has been supported by a Ph.D. scholarship from Thaksin University in Thailand for S. Amloy, grants from the Swedish Research Council (VR), the Swedish Foundation for Strategic Research (SSF) funded Nano-N consortium, and the Knut and Alice Wallenberg Foundation. The authors are thankful to P. Bergman for providing the time-resolved facilities.

${ }^{1}$ P. Michler, A. Kiraz, C. Becher, W. V. Schoenfeld, P. M. Petroff, L. Zhang, E. Hu, and A. Imamoğlu, Science 290, 2282 (2000).

${ }^{2}$ S. Kako, C. Santori, K. Hoshino, S. Götzinger, Y. Yamamoto, and Y. Arakawa, Nature Mater. 5, 887 (2006).

${ }^{3}$ P. Michler, A. Imamoğlu, M. D. Mason, P. J. Carson, G. F. Strouse, and S. K. Buratto, Nature 406, 968 (2000).

${ }^{4}$ C. Santori, S. Götzinger, Y. Yamamoto, S. Kako, K. Hoshino, and Y. Arakawa, Appl. Phys. Lett. 87, 051916 (2005).

${ }^{5}$ C. Kurtsiefer, P. Zarda, M. Halder, H. Weinfurter, P. M. Gorman, P. R. Tapster, and J. G. Rarity, Nature 419, 450 (2002).

${ }^{6}$ C. Z. Peng, T. Yang, X. H. Bao, J. Zhang, X. M. Jin, F. Y. Feng, B. Yang, J. Yang, J. Yin, Q. Zhang, N. Li, B. L. Tian, and J. W. Pan, Phys. Rev. Lett. 94, 150501 (2005).

${ }^{7}$ D. Loss and D. P. DiVincenzo, Phys. Rev. A 57, 120 (1998).

${ }^{8}$ S. De Rinaldis, I. D'Amico, E. Biolatti, R. Rinaldi, R. Cingolani, and F. Rossi, Phys. Rev. B 65, 081309R (2002).

${ }^{9}$ D. Simeonov, A. Dussaigne, R. Butté, and N. Grandjean, Phys. Rev. B 77, 075306 (2008).

${ }^{10}$ S. Amloy, K. H. Yu, K. F. Karlsson, R. Farivar, T. G. Andersson, and P. O. Holtz, Appl. Phys. Lett. 99, 251903 (2011). 
${ }^{11}$ F. Ding, R. Singh, J. D. Plumhof, T. Zander, V. Křápek, Y. H. Chen, M. Benyoucef, V. Zwiller, K. Dörr, G. Bester, A. Rastelli, and O. G. Schmidt, Phys. Rev. Lett. 104, 067405 (2010).

${ }^{12}$ R. Bardoux, A. Kaneta, M. Funato, Y. Kawakami, A. Kikuchi, and K. Kishino, Phys. Rev. B 79, 155307 (2009).

${ }^{13}$ S. Amloy, K. F. Karlsson, T. G. Andersson, and P. O. Holtz, Appl. Phys. Lett. 100, 021901 (2012).

${ }^{14}$ M. Winkelnkemper, R. Seguin, S. Rodt, A. Schliwa, L. Reißmann, A. Strittmatter, A. Hoffmann, and D. Bimberg, J. Appl. Phys. 101, 113708 (2007).

${ }^{15}$ R. Bardoux, T. Guillet, B. Gil, P. Lefebvre, T. Bretagnon, T. Taliercio, S. Rousset, and F. Semond, Phys. Rev. B 77, 235315 (2008).

${ }^{16}$ S. Amloy, Y. T. Chen, K. F. Karlsson, K. H. Chen, H. C. Hsu, C. L. Hsiao, L. C. Chen, and P. O. Holtz, Phys Rev. B 83, 201307(R) (2011).

${ }^{17}$ T. Bartel, M. Dworzak, M. Strassburg, A. Hoffmann, A. Strittmatter, and D. Bimberg, Appl. Phys. Lett. 85, 1946 (2004).

${ }^{18}$ J. W. Robinson, J. H. Rice, A. Jarjour, J. D. Smith, R. A. Taylor, R. A. Oliver, G. A. D. Briggs, M. J. Kappers, C. J. Humphreys, and Y. Arakawa, Appl. Phys. Lett. 83, 2674 (2003).

${ }^{19}$ T. Bretagnon, P. Lefebvre, P. Valvin, R. Bardoux, T. Guillet, T. Taliercio, B. Gil, N. Grandjean, F. Semond, B. Damilano, A. Dussaigne, and J. Massies, Phys. Rev. B 73, 113304 (2006).

${ }^{20}$ M. Winkelnkemper, M. Dworzak, T. P. Bartel, A. Strittmatter, A. Hoffmann, and D. Bimberg, Phys. Status Solidi B 245, 2766 (2008).

${ }^{21}$ K. Sebald, J. Kalden, H. Lohmeyer, and J. Gutowski, Phys. Status Solidi B 248, 1777 (2011).
${ }^{22}$ J. H. Rice, J. W. Robinson, J. H. Na, K. H. Lee, R. A. Taylor, D. P. Williams, E. P. O'Reilly, A. D. Andreev, Y. Arakawa, and S. Yasin, Nanotechnology 16, 1477 (2005).

${ }^{23}$ K. Brunner, G. Abstreiter, G. Böhm, G. Tränkle, and G. Weimann, Phys. Rev. Lett. 73, 1138 (1994).

${ }^{24}$ C. W. Hsu, E. S. Moskalenko, M. Eriksson, A. Lundskog, K. F. Karlsson, U. Forsberg, E. Janzén and P. O. Holtz, "The negatively-charged exciton in an InGaN quantum dot on a GaN pyramid" (unpublished).

${ }^{25}$ K. F. Karlsson, E. S. Moskalenko, P. O. Holtz, B. Monema, W. V. Schoenfeld, J. M. Garcia, and P. M. Petroff, Appl. Phys. Lett. 78, 2952 (2001).

${ }^{26}$ E. S. Moskalenko, K. F. Karlsson, P. O. Holtz, B. Monemar, W. V. Schoenfeld, J. M. Garcia, and P. M. Petroff. J. Appl. Phys. 92, 6787 (2002).

${ }^{27}$ G. Bacher, R. Weigand, J. Seufert, V. D. Kulakovskii, N. A. Gippius, A. Forchel, K. Leonardi, and D. Hommel, Phys. Rev. Lett. 83, 4417 (1999).

${ }^{28}$ H. Gotoh, T. Akasaka, T. Tawara, Y. Kobayashi, T. Makimoto, and H. Nakano, Solid State Commun. 138, 590 (2006).

${ }^{29}$ M. Sénès, K. L. Smith, T. M. Smeeton, S. E. Hooper, and J. Heffernan, Phys. Rev. B 75, 045314 (2007).

${ }^{30}$ H. Gotoh, T. Tawara, Y. Kobayashi, N. Kobayashi, and T. Saitoh, Appl. Phys. Lett. 83, 4791 (2003).

${ }^{31}$ M. Feucker, R. Seguin, S. Rodt, A. Hoffmann, and D. Bimberg, Appl. Phys. Lett. 92, 063116 (2008).

${ }^{32}$ R. M. Thompson, R. M. Stevenson, A. J. Shields, I. Farrer, C. J. Lobo, D. A. Ritchie, M. L. Leadbeater, and M. Pepper, Phys. Rev. B 64, 201302R (2001). 